Document downloaded from:

http://hdl.handle.net/10251/160420

This paper must be cited as:

Conejero, JA.; Lizama, C.; Mira-Iglesias, A.; Rodero-Gómez, C. (2019). Visibility graphs of fractional Wu-Baleanu time series. The Journal of Difference Equations and Applications. 25(9-10):1321-1331. https://doi.org/10.1080/10236198.2019.1619714

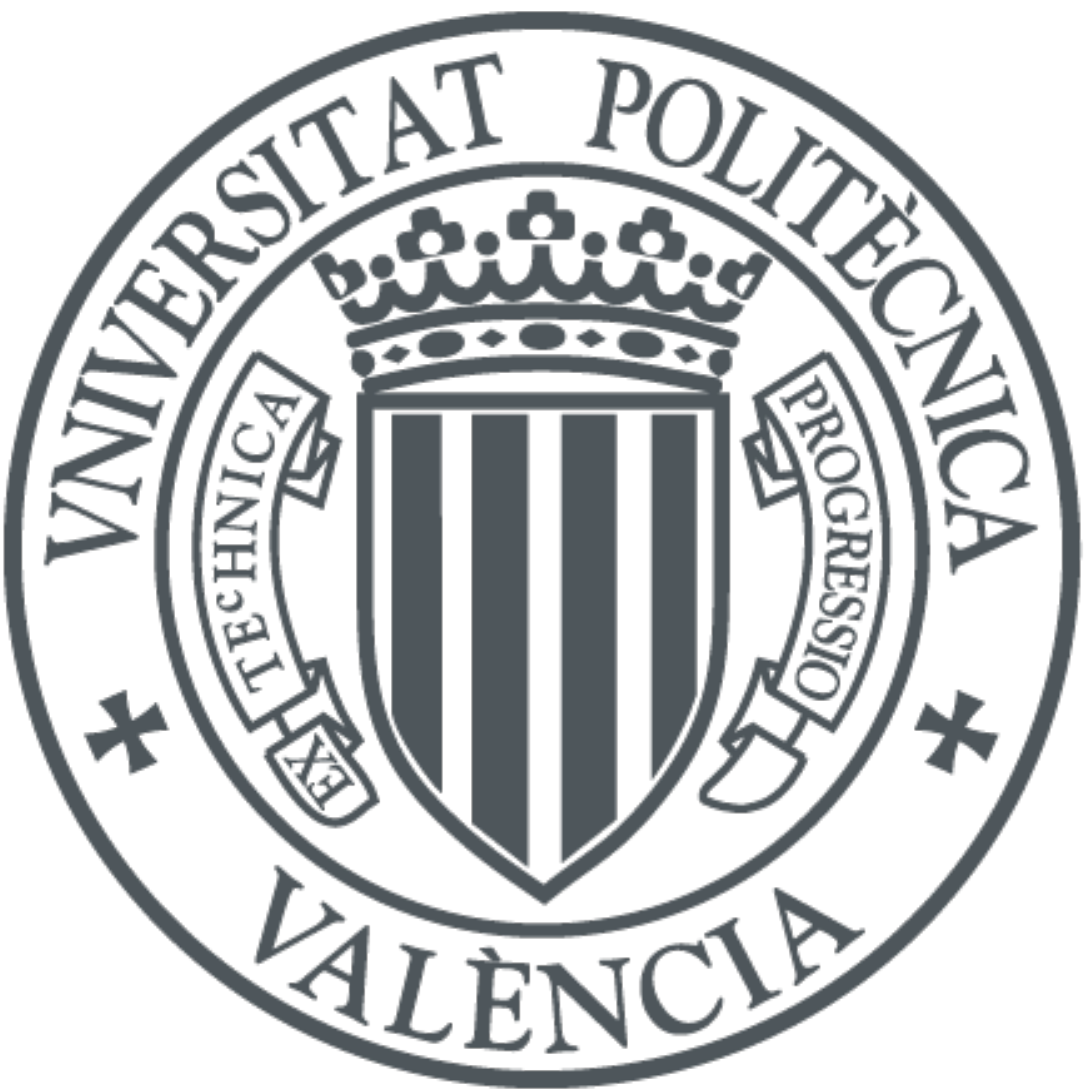

The final publication is available at

https://doi.org/10.1080/10236198.2019.1619714

Copyright Taylor \& Francis

Additional Information 


\title{
Visibility graphs of fractional Wu-Baleanu time series
}

\author{
J. Alberto Conejero ${ }^{\mathrm{a}}$, Carlos Lizama ${ }^{\mathrm{b}}$, Ainara Mira-Iglesias ${ }^{\mathrm{c}}$, Cristóbal \\ Rodero-Gómez d \\ anstituto Universitario de Matemática Pura y Aplicada, Universitat Politècnica de València, \\ E46022, València, Spain. \\ ${ }^{\mathrm{b}}$ Universidad de Santiago de Chile, Facultad de Ciencias, Departamento de Matemática y \\ Ciencia de la Computación, Las Sophoras 173, Santiago, Chile. \\ ${ }^{\mathrm{c}}$ Fundación para el Fomento de la Investigación Sanitaria y Biomédica de la Comunitat \\ Valenciana (FISABIO), Valencia, Spain. \\ ${ }^{\mathrm{d} D i v i s i o n}$ of Imaging Sciences and Biomedical Engineering, King's College London, St. \\ Thomas' Hospital, SE1 7EH London, United Kingdom.
}

\section{ARTICLE HISTORY}

Compiled May 9, 2019

\begin{abstract}
We study time series generated by the parametric family of fractional discrete maps introduced by $\mathrm{Wu}$ and Baleanu in [34], presenting an alternative way of introducing these maps.

For the values of the parameters that yield chaotic time series, we have studied the Shannon entropy of the degree distribution of the natural and horizontal visibility graphs associated to these series. In these cases, the degree distribution can be fitted with a power law. We have also compared the Shannon entropy and the exponent of the power law fitting for the different values of the fractionary exponent and the scaling factor of the model. Our results illustrate a connection between the fractionary exponent and the scaling factor of the maps, with the respect to the onset of the chaos.
\end{abstract}

\section{KEYWORDS}

Discrete fractional calculus; Caputo delta difference; logistic equation; visibility graphs.

\section{AMS CLASSIFICATION \\ 37B10,39A06,26A33.}

\section{Introduction}

The classical logistic map popularized by May [24] is given by

$$
v(n+1)=\mu v(n)(1-v(n))
$$

where $v(0) \in[0,1]$ and $\mu \in \mathbb{R}$. When $0 \leq \mu \leq 4$, the logistic equation (1) gives a discrete dynamical system defined on $[0,1]$. For cases $\mu>4$, we have a discrete dynamical system defined on the complementary of a certain Cantor set in $[0,1]$. The dynamics of a one-parameter discrete dynamical system is usually represented by a 
Feigenbaum (bifurcation) diagram. A point $(\bar{\mu}, \bar{v})$ in this diagram represents that when we take the parameter $\bar{\mu}, \bar{v}$ is in the $\omega$-limit of a particular initial condition.

$\mathrm{Wu}$ and Baleanu recently studied the chaos of a discrete fractional logistic map inspired in the logistic equation from the left Caputo discrete delta difference [34]. They first showed an approach to how the Feigenbaum diagrams evolve when the tuner $\mu$ of the fractional derivative varies. The delayed version of these models has been studied by the same authors in [33].

Besides those diagrams, other techniques can provide some insight into the dynamics of the discrete fractional logistic model. In this work, we consider the use of natural and horizontal visibility graphs associated with time series generated by iteration under the Wu-Baleanu model. They were introduced by Lacasa et al. in [18] and by Luque et al. in [21] in order to analyse the evolution of a time series by mapping it into a graph. They showed that the degree distribution in these graphs is inherited from the structure of the given time series. For instance, periodic series are converted into regular graphs, random series into random graphs and fractality is associated with a power law distribution of the degree frequencies in these graphs. As it was noted by Barabási and Albert, power law distributions for the values of the degrees are associated with scale-free networks $[4,5]$.

This approach belongs to an emerging corpus of methods that map series to networks, see for instance $[8,16,35]$. Applications of such time series analysis can be found in climate dynamics, multiphase flow, brain functions, ECG dynamics, economics and traffic systems [12].

The degree distribution of the nodes of the associated networks usually follows a power law distribution. Its connection with chaotic maps, flows, and stochastic processes were shown in [27]. The study of Feigenbaum diagrams of the logistic map and its connection with horizontal visibility graphs has been already considered in [22]. In this direction, these authors introduced what they call Feigenbaum graphs in order to represent the dynamics of all stationary trajectories of this map. Moreover, they showed that the network entropy mimics the Lyapunov exponent of the map independently of its sign. See [15] for more methods and metrics for carrying out this kind of analysis. See also [32] for a revision of different approximations to entropy for network parameters.

In this work, we are going to consider the use of natural and horizontal visibility graphs associated to time series generated by the discrete fractional model formerly introduced from the fractional logistic map in [34]. We also study the links between the evolution of the scaling factor and fractional exponent with the exponent used when fitting the distribution of degrees of visibility graphs to power laws distributions. This provides an integrated vision of the dynamics that cannot be obtained from single Feigenbaum diagrams computed for every single case.

In Section 2 we introduce the fractional discrete model under study. We propose an alternative way of introducing it respect to the one shown by $\mathrm{Wu}$ and Baleanu in [34]. In Section 3, we recall some basic facts from visibility graphs. An analysis of the dynamics of these fractional maps in terms of visibility graphs is shown in Section 4. We also illustrate the connection of the power law exponent with the Shannon entropy of the degree distribution of the visibility graphs. Finally, conclusions are outlined in Section 5 . 


\section{Discrete fractional logistic model}

Fractional integrals and derivatives are extensions of the classical ones and they are the ideal candidates for modelling processes that contain some memory (spatial or temporal). The following difference equation

$$
\Delta u(n)=\mu u(n)(1-u(n)) \quad u(0)=u_{0},
$$

where $\Delta$ denotes the forward Euler operator, can be transformed into a logistic equation with the change of variable $v(n)=\frac{\mu}{\mu+1} u(n)$, which yields

$$
v(n+1)=(1+\mu) v(n)(1-v(n)), \quad v(0)=\frac{\mu}{1+\mu} u(0) .
$$

The next definition was presented in [19, Formula 2.2] in the context of abstract Cauchy problems on time difference equations. It corresponds to a particular case of the fractional sum proposed by Atici and Eloe in [2]. It has been recently observed that it coincides with the notion of Cesàro sums of order $\alpha>0$ and possesses a strong connection with certain algebra homomorphisms.

Definition 2.1. Let $\alpha>0$ and $u: \mathbb{N}_{0} \rightarrow X$. We define the fractional sum of order $\alpha$ as follows

$$
\Delta^{-\alpha} f(n)=\sum_{k=0}^{n} k^{\alpha}(n-k) u(k), \quad n \in \mathbb{N}_{0},
$$

where

$$
k^{\alpha}(j):=\frac{\Gamma(\alpha+j)}{\Gamma(\alpha) \Gamma(j+1)}, \quad j \in \mathbb{N}_{0} .
$$

The numbers $k^{\alpha}(n)$ are called Cesàro numbers of order $\alpha$ ([36, Vol. I, p.77]). The kernels $k^{\alpha}$ may equivalently be defined by means of the generating functions:

$$
\sum_{n=0}^{\infty} k^{\alpha}(n) z^{n}=\frac{1}{(1-z)^{\alpha}}, \quad|z|<1, \quad \alpha>0 .
$$

It can be checked that the numbers $k^{\alpha}(n)$ satisfy the semigroup property, that is, $k^{\alpha} * k^{\beta}=k^{\alpha+\beta}$ for $\alpha, \beta>0$. Moreover, for every $\alpha>0$ the following equality holds:

$$
k^{\alpha}(n)=\frac{n^{\alpha-1}}{\Gamma(\alpha)}\left(1+\mathcal{O}\left(\frac{1}{n}\right)\right), \quad n \in \mathbb{N}
$$

([36, Vol. I, p.77 (1.18)]). For $\alpha>1$, the kernel $k^{\alpha}$ is increasing (as a function of $n$ ), for $0<\alpha<1$ it is decreasing, and $k^{1}(n)=1$ for $n \in \mathbb{N}_{0}$ ([36, Theorem III.1.17]). It is also straightforward to check that $k^{\alpha}(n) \leq k^{\beta}(n)$ for $0<\alpha \leq \beta$ and $n \in \mathbb{N}_{0}$.

Remark 1. We note that allowing $\alpha<0$ in the above definition, we get the concept of fractional difference in the sense of Grünwald-Letnikov. Observe that this definition 
corresponds to a discretization using the forward Euler operator, see the comments after Definition 2.1 in [19]. Details of the numerical computation of fractional derivatives can be found in $[6,7]$.

The following notion is similar to the concept of a fractional derivative in the sense of Riemann-Liouville, see [25] and [3]. It is interesting to observe that it coincides with the sampling obtained with the Poisson distribution of the continuous fractional difference of the same order. See [20, Theorem 3.5].

Definition 2.2. [20] Given $f: \mathbb{N}_{0} \rightarrow X$, the fractional difference operator of order $\alpha>0$ (in the sense of Riemann-Liouville) is defined by

$$
\Delta^{\alpha} f(n):=\Delta^{m} \circ \Delta^{-(m-\alpha)} f(n), \quad n \in \mathbb{N}_{0},
$$

where $m-1<\alpha<m, m=\lceil\alpha\rceil$.

Interchanging the order of the operators in the definition of fractional difference in the sense of Riemann-Liouville, the notion of fractional difference in the sense of Caputo is defined as follows.

Definition 2.3. [20] Let $f: \mathbb{N}_{0} \rightarrow X$ and $\alpha>0$. The $\alpha$-th fractional Caputo like difference is defined by

$$
{ }_{C} \Delta^{\alpha} f(n):=\Delta^{-(m-\alpha)}\left(\Delta_{0}^{m} f\right)(n), \quad n \in \mathbb{N}_{0},
$$

where $m-1<\alpha<m, m=\lceil\alpha\rceil$.

Recall that the finite convolution $*$ of two sequences $f(n)$ and $g(n)$ is defined by

$$
(f * g)(n):=\sum_{j=0}^{n} f(n-j) g(j), \quad n \in \mathbb{N}_{0} .
$$

For further use, we note the following relation between the Caputo and RiemannLiouville fractional differences of order $0<\alpha<1$.

Theorem 2.4. [19, Theorem 2.9] For each $0<\alpha<1$ and $u \in s\left(\mathbb{N}_{0} ; X\right)$, we have

$$
{ }_{C} \Delta^{\alpha} u(n)=\Delta^{\alpha} u(n)-k^{1-\alpha}(n+1) u(0), \quad n \in \mathbb{N}_{0} .
$$

Let us fix some $0<\nu<1$. We consider the fractional analogous of $(2)$

$$
{ }_{C} \Delta^{\nu} u(t)=\mu u(n)(1-u(n)),
$$

with $u(0)=u_{0}$.

Using the property $\Delta^{-\nu}{ }_{C} \Delta^{\nu} f(n)=f(n+1)-f(0)$, the discrete fractional equation (10) can be rewritten as

$$
u(n)=u(0)+\mu \sum_{j=1}^{n} k^{\nu}(n-j)(u(j-1)(1-u(j-1)) .
$$


In comparison with (1), the model in (11) includes some memory, since the value at $u(n)$, depends on the previous past values $u(i)$, with $0 \leq i \leq n-1$. It also depends on a parameter $\nu$.

Remark 2. It is notable that the above expression coincides with those proposed by Wu and Baleanu in [34, Formula (14)]. However, this connection has not been observed before.

\section{Visibility graphs}

Visibility algorithms have been proposed in order to study time series as complex networks. This allows to characterize the process in terms of network science. The key idea is to convert the time series into a network, and then try to determine whether this network inherits some structure of the time series. Given a time series $\left\{\left(n, u_{n}\right)\right.$ : $n \in \mathbb{N}\}$, two visibility graphs have been considered:

NVG. The Natural Visibility Graph was introduced in [18]. Here, a node corresponds to each data in the time series. The correspondence is set in the same order as it appears in the time series. Two nodes are connected by an edge if there is a straight line that connects both nodes, and this line does not intersect any intermediate data height. That is, nodes of any two arbitrary values $\left(n_{a}, y_{a}\right)$ and $\left(n_{b}, y_{b}\right)$ are connected by an edge if any other data $\left(n_{c}, y_{c}\right)$, with $n_{a}<n_{c}<n_{b}$, fulfills:

$$
y_{c}<y_{b}+\left(y_{a}-y_{b}\right) \frac{n_{b}-n_{c}}{n_{b}-n_{a}} .
$$

See the left part of figure 1 for a visual interpretation.

HVG. Horizontal Visibility Graph was later introduced in [21]. It is a particular case of NVG. Here, nodes associated to $\left(n_{a}, y_{a}\right)$ and $\left(n_{b}, y_{b}\right)$ are connected by an edge if for any other node $n_{a}<n_{c}<n_{n}$, we have:

$$
y_{a}>y_{c} \text { and } y_{b}>y_{c} \text {. }
$$

See the right part of figure 1 for a visual interpretation.

As a matter of fact, analysis of degree distributions, clustering coefficients, and mean path length of NVG's and HVG's permits to identify when randomness is present, see for instance $[17,18,21,22]$.

The resulting networks are always connected and have interesting properties, such as the invariance of the graph under affine transformations on the data series. NVG's and HVG's capture the hub repulsion phenomenon associated with fractal networks [30] and thus distinguishes scale-free visibility graphs evidencing the small-world effect from those showing scale invariance. More precisely, periodic series are converted into regular networks, random series into random networks, and fractal series into scale-free networks.

Chaotic series from logistic and Hénon maps have been compared and distinguished from independent and identically distributed random variables in [21, 22]. HVG's associated with any random series give small-world random graphs with a universal exponential degree distribution of the form $P(k)=\frac{1}{3}(2 / 3)^{k-2}$, independently of the 

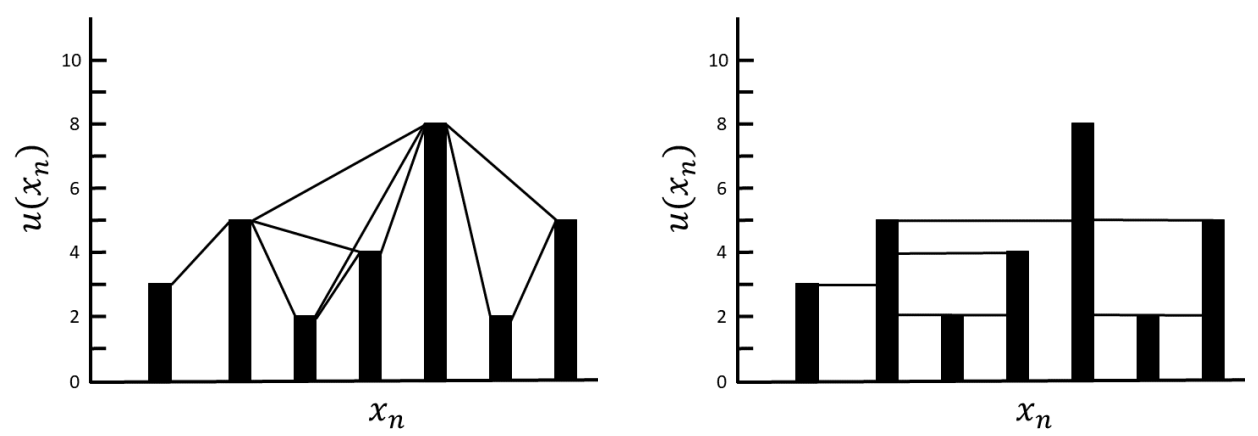

Figure 1. Determination of links for the construction of the NVG (left) and HVG (right).

probability distribution. This provides an easy test for randomness. A more-in-depth study of the different approaches to chaos has also been considered with the use of HVG's over trajectories obtained with the logistic maps. Different phenomena already studied comprise the period-doubling bifurcation cascade [22], the quasiperiodicity [23], and the intermittency, that is the seemingly random alternation of long laminar phases, and relatively short chaotic bursts [26]. These approaches have been revisited in [28].

In fact, new phenomena have arisen in the light of the visibility graphs approach, such as sequential visibility graph motifs, smaller substructures of $n$ consecutive nodes that appear with characteristic frequencies [13].

Further analyses can be performed on the degree distribution of the visibility graphs, such as the computation of Shannon entropy [29]. The entropy $h$ of a discrete random variable $X$ is calculated as

$$
h(X, \Omega)=-\sum_{x_{i} \in \Omega} p\left(x_{i}\right) \log _{2} p\left(x_{i}\right),
$$

where $\Omega$ is certain set where $X$ is defined, and $p\left(x_{i}\right)$ is the probability that $X=x_{i}$. In the case of the visibility graphs, it can be rewritten as

$$
h\left(n_{i}\right)=-\sum_{j=1}^{\infty} p_{j}\left(n_{i}\right) \log _{2}\left(p_{j}\left(n_{i}\right)\right),
$$

where $p_{j}\left(n_{i}\right)$ is the probability that the node $i$ has degree $j$ - although in some cases the base 2 logarithm has been change for the natural logarithm. More information on the quantification of the complexity of a network can be found in [1]. Worth to mention are the Lyapunov exponents, which highly correlate with the entropy as Luque et al. showed in [22]. For this reason we have decided to restrict ourselves to the entropy instead of the Lyapunov exponents in this work. 


\section{Results}

\subsection{Time series analyses}

The logistic equation (1) starts to exhibit chaos from approximately 3.57 until 4, as it can be observed from the bifurcation diagrams, see for instance [31]. Wu and Baleanu also used Feigenbaum diagrams in order to numerically illustrate chaos phenomena for particular cases of the fractionary exponent $\nu$ and the scaling factor $\mu$ of the model. In fact, just a change in the values of $\nu$ in (11) lets us appreciate chaotic phenomena in different zones, see [34, Fig. 4 \& Fig. 7].

In figure 2, we have recalculated these diagrams with higher resolution. These diagrams were generated starting with $u(0)=0.3$ as initial condition and with a step size for $\mu$ of 0.001 in all the cases. For every pair of values of $\nu$ and $\mu$ we have computed 200 iterations, considering the last 100 of them to be plotted.
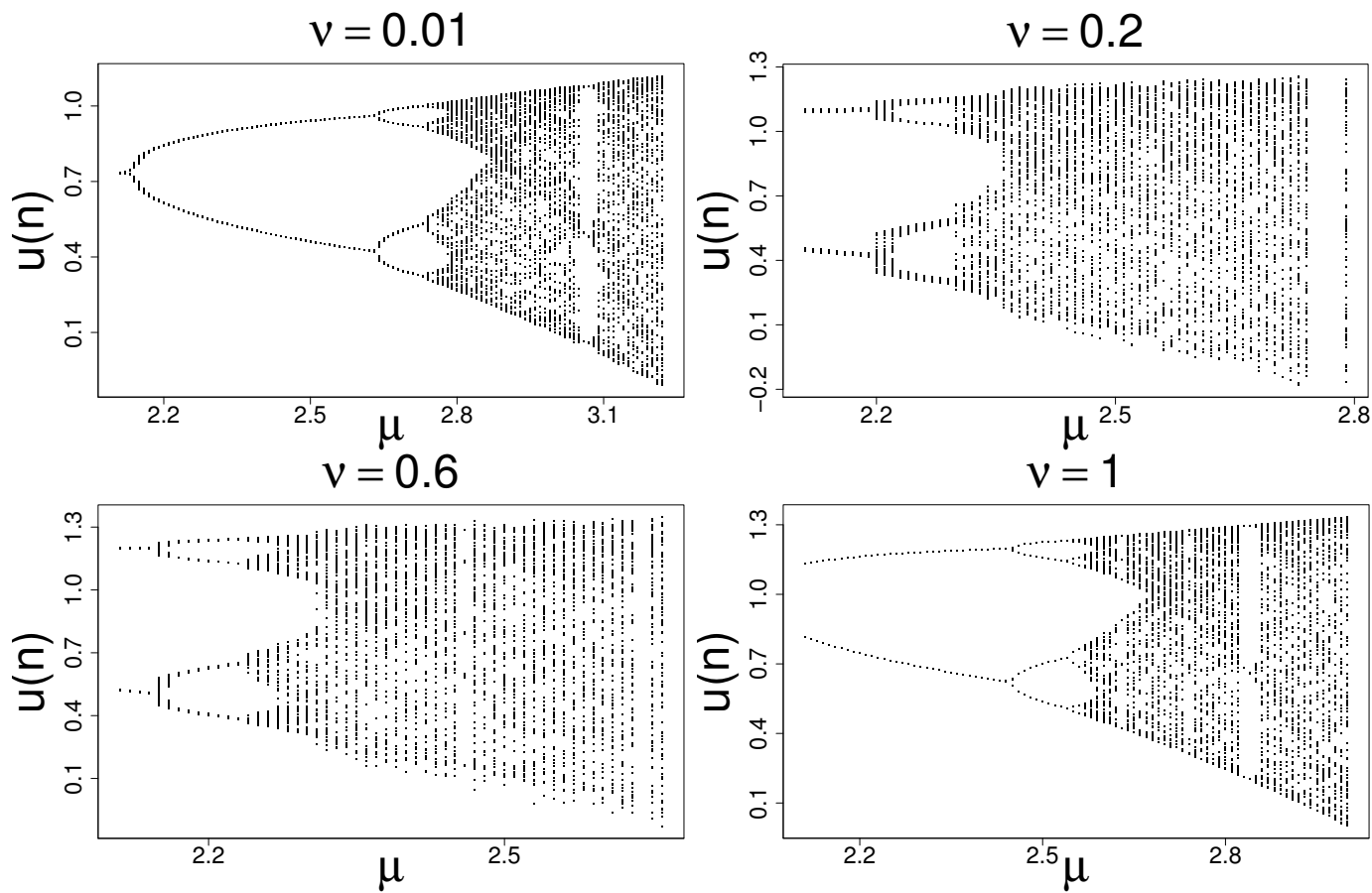

Figure 2. Feigenbaum diagrams for the Wu-Baleanu model: Top-left $\nu=0.01$ and $2.1 \leq \mu \leq 3$ and $3.1 \leq$ $\mu \leq 3.20$; top right $\nu=0.2$ and $2.39 \leq \mu \leq 2.62$, bottom-left $\nu=0.6$ and $2.35 \leq \mu \leq 2.66$, bottom-right $\nu=1$ and $2.70 \leq \mu \leq 3.00$.

When analysing these diagrams it is not clear where to establish the threshold of the onset of the chaos. This uncertainty is due to the coarseness of the plot since a closer look into the zone of interest would show another duplication of the orbits in the $\omega$-limit, and so on until the points in the bifurcation diagram are too entangled to distinguished one limit from another. Improving the number of iterations and the parameter step, these could be recognized. For more detailed studies of chaos of fractional discrete maps we refer to [9-11]. 


\subsection{Visibility graphs analysis}

As it has been previously discussed in Section 3, both NVG's and HVG's are related to a single time series, i.e. a single value of $\mu$ in the Feigenbaum diagrams. A tool to be able to measure chaos in a more quantifiable way than with these diagram is through the computation of the Shannon entropy applied to the visibility graphs derived from time series. We have calculated the Shannon entropy for different pairs $(\mu, \nu)$ in $(\mu, \nu) \in[2.1,3.3] \times[0,1]$, with a step of 0.01 for both parameters. For each pair, we have then computed the exponent of the power law fitting to the degree distribution as it has been explained in Section 3.

Shannon entropy of the NVG associated to each pair $(\mu, \nu)$ is illustrated in figure 3 through a heat map. It can be appreciated that there exists a clear pattern of chaos across the parametric space. A symmetry with respect to $\nu \approx 0.4$ is noted, as well as a Hénon map-like shape. Moreover, there seems to be non-gradual steps of entropy change, following the same approximate shape but in at least two clearly different zones (light blue-yellow in figure 3). The blank space in the right part of the figure is due to the divergence of the time series. In general trends, the more fractional the equation is (closer to $\nu=0.5$ ) the sooner the chaos onset appears for lower values of the scaling factor $\mu$.

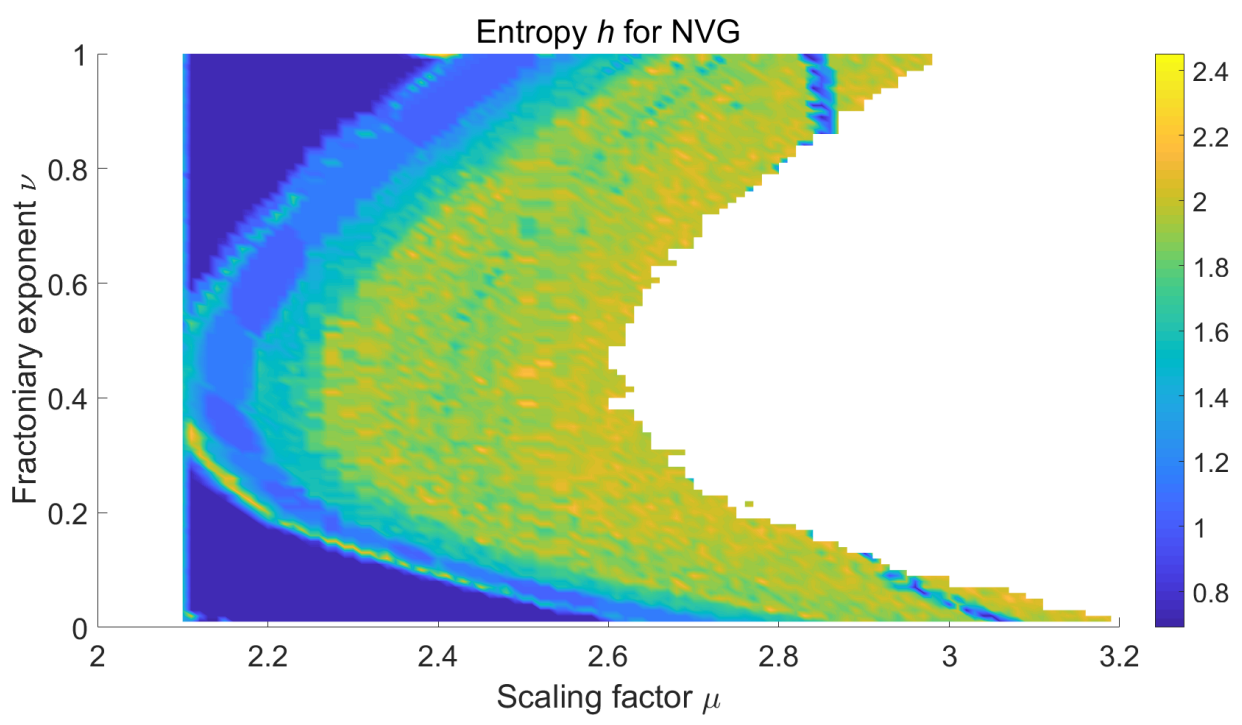

Figure 3. Shannon entropy for the different values of the Wu-Baleanu scaling factor $\mu$ and the fractionary exponent $\nu$ in the case of the natural visibility graphs.

Analogously, the entropy heat map for the HVG Shannon entropies is shown in figure 4. A quick visual inspection of this plot compared with figure 3 reveals high similarities between both of them, including the different C-shaped waves of entropy increment. In order to quantify these similarities, we have computed several correlation metrics for matrices between the HVG's matrix and the NVG's matrix, already considered in the literature [14]. The results can be observed in the third column of table 1 . With such high correlation values, we can state that the information concerning the chaos onset from the NVG's is essentially the same present in the HVG's.

Finally, we have computed the exponents of the power law fitting of the degree distribution of the NVG's for each combination of $(\mu, \nu)$. The results can be observed in figure 5 . This resembles the aforementioned entropy heat maps, with a more clear gra- 


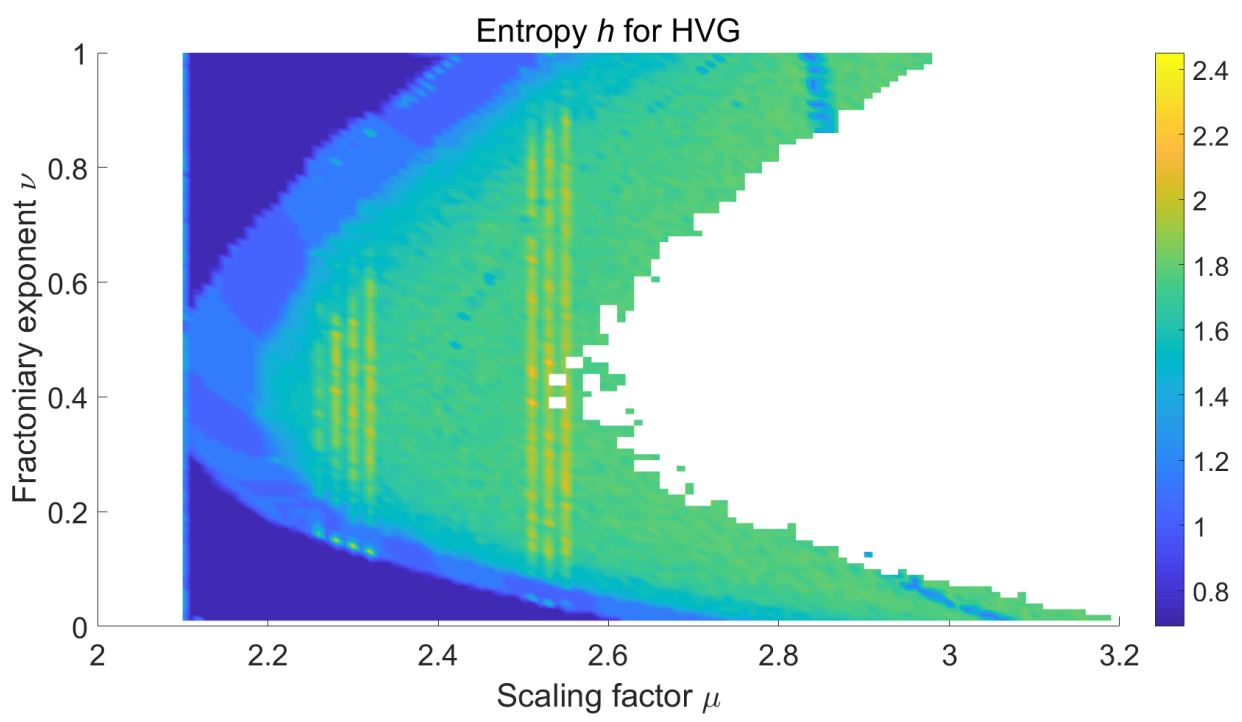

Figure 4. Shannon entropy for the different values of the Wu-Baleanu scaling factor $\mu$ and the fractionary exponent $\nu$ in the case of the horizontal visibility graphs.

\begin{tabular}{|c|c|c|c|}
\hline Correlation matrices & $c\left(\mathbf{X}_{1}, \mathbf{X}_{2}\right)$ formula & $c\left(M_{\mathrm{NVG}}, M_{\mathrm{HVG}}\right)$ & $c\left(M_{\mathrm{NVG}}, M_{\mathrm{exp}}\right)$ \\
\hline$S M I_{\mathrm{OP}}$ & $\frac{\left\|\mathbf{X}_{1}^{T} \mathbf{X}_{2}\right\|_{F}^{2}}{\min (p, q)}$ & 1.000 & 1.000 \\
\hline$R V$ & $\begin{array}{c}\frac{\operatorname{tr}\left(\mathbf{Y}_{1}^{T} \mathbf{Y}_{2}\right)}{\left(\operatorname{tr}\left(\mathbf{Y}_{1}^{T} \mathbf{Y}_{1}\right) \operatorname{tr}\left(\mathbf{Y}_{2}^{T} \mathbf{Y}_{2}\right)\right)^{1 / 2}} \\
\mathbf{Y}_{1}=\mathbf{X}_{1} \mathbf{X}_{1}^{T}, \mathbf{Y}_{2}=\mathbf{X}_{2} \mathbf{X}_{2}^{T}\end{array}$ & 0.999 & 0.984 \\
\hline$R V 2$ & $\begin{array}{c}\frac{\operatorname{tr}\left(\mathbf{Y}_{1}^{T} \mathbf{Y}_{2}\right)}{\left(\operatorname{tr}\left(\mathbf{Y}_{1}^{T} \mathbf{Y}_{1}\right) \operatorname{tr}\left(\mathbf{Y}_{2}^{T} \mathbf{Y}_{2}\right)\right)^{1 / 2}} \\
\mathbf{Y}_{1}=\mathbf{X}_{1} \mathbf{X}_{1}^{T}-\operatorname{diag}\left(\mathbf{X}_{1} \mathbf{X}_{1}^{T}\right), \mathbf{Y}_{2}=\mathbf{X}_{2} \mathbf{X}_{2}^{T}-\operatorname{diag}\left(\mathbf{X}_{2} \mathbf{X}_{2}^{T}\right)\end{array}$ & 0.999 & 0.984 \\
\hline$R V_{a d j}$ & $\begin{array}{c}\frac{p \cdot q \cdot n_{c}+n_{r} \cdot \operatorname{tr}\left(\mathbf{C}_{12}^{T} \mathbf{C}_{12}\right)}{\left(\left[p^{2} \cdot n_{c}+n_{r} \cdot \operatorname{tr}\left(\mathbf{C}_{11}^{T} \mathbf{C}_{11}\right)\right]\left[q^{2} \cdot n_{c} \cdot \operatorname{tr}\left(\mathbf{C}_{22}^{T} \mathbf{C}_{22}\right)\right)^{1 / 2}\right.} \\
n_{r}=(n-1) /(n-2), n_{c}=1-n_{r}\end{array}$ & 0.999 & 0.984 \\
\hline$P S I$ & Average of the non-zero singular values of $\mathbf{X}_{1}^{T} \mathbf{X}_{2}$. & 0.993 & 0.971 \\
\hline$G C D$ & $\begin{array}{l}R V(\mathbf{T U}), \text { where } \mathbf{T} \text { and } \mathbf{U} \text { are orthogonal bases } \\
\text { for the column spaces of } \mathbf{X}_{1} \text { and } \mathbf{X}_{2} \text {, respectively. }\end{array}$ & 1.000 & 1.000 \\
\hline
\end{tabular}

Table 1. Different correlation metrics $c(\cdot, \cdot)$ for matrices [14] computed for the NVG matrix $M_{\mathrm{NVG}}$ and the HVG matrix $M_{\mathrm{HVG}}$ (3rd column), and for the $M_{\mathrm{NVG}}$ and the matrix with the exponents of the power law fitting $M_{\exp }$ (4th column). Explanation of the formulae (2nd column): $\mathbf{X}^{T}$ is the transpose of $\mathbf{X},\|\cdot\|_{F}$ stands for the Fröbenius norm, $p$ is the number of columns of $\mathbf{X}_{1}, q$ is the number of columns of $\mathbf{X}_{2}, \operatorname{tr}(\cdot)$ is the trace, $\operatorname{diag}(\cdot)$ is the matrix diagonal and $C_{i j}$ is the correlation matrix between $\mathbf{X}_{i}$ and $\mathbf{X}_{j}$.

dient in the first C-shaped wave of the chaos onset. In the fourth column of Table 1 the different correlations defined in [14] have been computed between the NVG's entropy matrix and the power law exponent fitting matrix. As it happened with both visibility graphs matrices, the correlations are high in all the cases, meaning that the chaosrelated information encoded by the exponent of the power law fitting is qualitatively the same as within the entropy of the NVG's.

\section{Conclusions}

In this work, we have analyzed the chaos for a parametric family of fractional discrete maps. We have tried to describe the chaos phenomena in terms of the Shannon entropy of natural and horizontal visibility graphs associated with time series obtained from these models. We have also compared these results with the exponent of the power law fitting of each time series. 


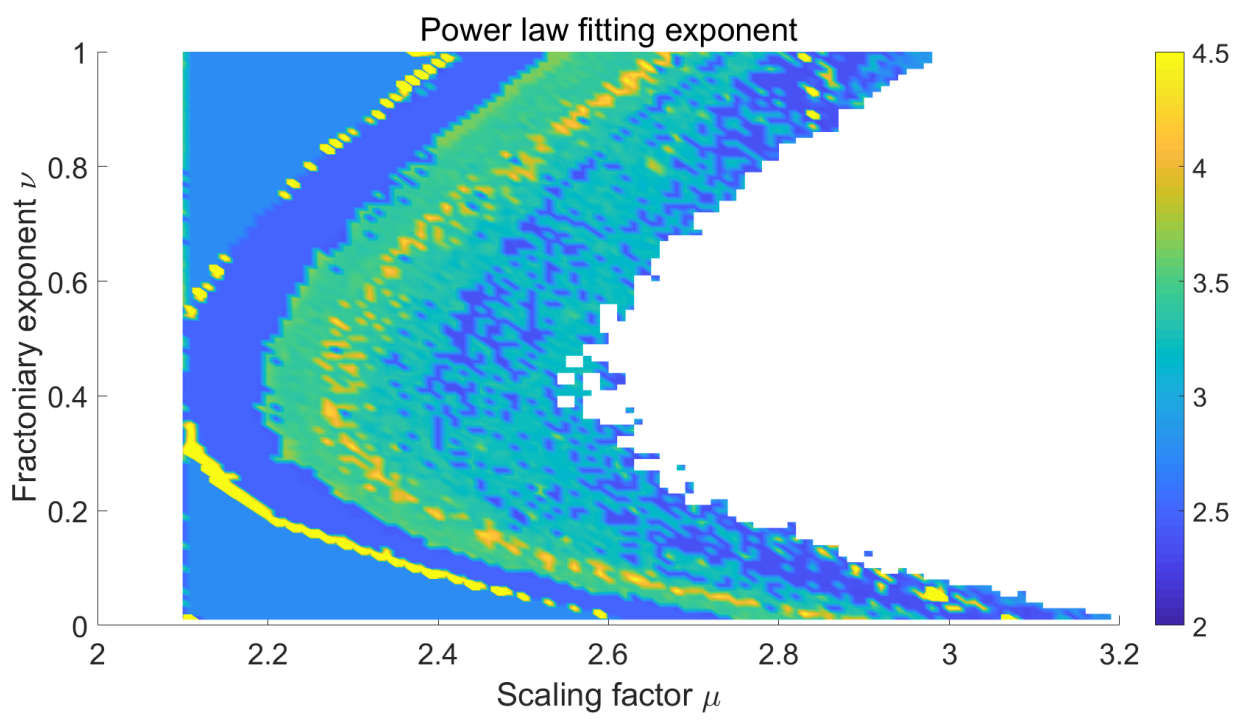

Figure 5. Exponents for the power law fitting of the the natural visibility graphs degree distribution for the different combinations of the Wu-Baleanu scaling factor $\mu$ and the fractional exponent $\nu$. Values over 5 have been disregarded of the plot, for the sake of visual comparison (these values where approximately $1.73 \%$ of the total amount of numerical values).

Some C-shape curves are exhibited showing the connections between the fractional exponent $\nu$ and the scaling factor $\mu$. In addition, there is also a region in which the orbits diverge and then the computation of the visibility graphs would not correctly describe such behaviour. It would be interesting if some insight could be provided into how both parameters $\nu$ and $\mu$ are linked. Besides, it will also be interesting to determine beforehand the borders of the region where there exists the $\omega$-limit of the orbits.

\section{Funding}

J.A. Conejero is supported MEC Grant Project MTM2016-75963-P. Carlos Lizama is supported by CONICYT, under Fondecyt Grant number 1180041. Cristóbal RoderoGómez is funded by EUs H2020 research and Innovation programme under the Marie Sklodowska-Curie grant agreement No 764738.

\section{References}

[1] K. Anand and G. Bianconi, Entropy measures for networks: Toward an information theory of complex topologies, Phys. Rev. E 80 (2009), p. 045102.

[2] F.M. Atıcı and P.W. Eloe, Discrete fractional calculus with the nabla operator, Electron. J. Qual. Theo. 2009 (2009), pp. 1-12.

[3] F. Atici and P. Eloe, A transform method in discrete fractional calculus, Int. J. Difference Equ. 2 (2007), pp. 165-176.

[4] A. Barabási, Network science, Cambridge University Press (2016).

[5] A. Barabási and R. Albert, Emergence of scaling in random networks, Science 286 (1999), pp. 509-512. 
[6] D.W. Brzeziński, Comparison of fractional order derivatives computational accuracy right hand vs left hand definition, Appl. Math. Nonlinear Sci. 2 (2017), pp. 237-248.

[7] D.W. Brzeziński, Review of numerical methods for NumILPT with computational accuracy assessment for fractional calculus, Appl. Math. Nonlinear Sci. 3 (2018), pp. 487-502.

[8] R. Donner, M. Small, J. Donges, N. Marwan, Y. Zou, R. Xiang, and J. Kurths, Recurrencebased time series analysis by means of complex network methods, Int. J. Bifurc. Chaos Appl. Sci. Eng. 21 (2011), pp. 1019-1046.

[9] M. Edelman, Fractional maps as maps with power-law memory, in Nonlinear dynamics and complexity, Springer, 2014, pp. 79-120.

[10] M. Edelman, On the fractional eulerian numbers and equivalence of maps with long term power-law memory (integral volterra equations of the second kind) to Grünvald-Letnikov fractional difference (differential) equations, Chaos 25 (2015), p. 073103.

[11] M. Edelman, On stability of fixed points and chaos in fractional systems, Chaos 28 (2018), p. 023112.

[12] Z.K. Gao, M. Small, and J. Kurths, Complex network analysis of time series, EPL (Europhysics Letters) 116 (2016), p. 50001.

[13] J. Iacovacci and L. Lacasa, Sequential visibility-graph motifs, Phys. Rev. E 93 (2016), p. 042309.

[14] U.G. Indahl, T. Næs, and K.H. Liland, A similarity index for comparing coupled matrices, J. Chemom. 32 (2018), p. e3049.

[15] H. Kantz and T. Schreiber, Nonlinear Time Series Analysis, 2nd ed., Cambridge University Press (2003).

[16] F. Kyriakopoulos and S. Thurner, Directed network representation of discrete dynamical maps, in International Conference on Computational Science. Springer (2007) pp. 625632.

[17] L. Lacasa and J. Iacovacci, Visibility graphs of random scalar fields and spatial data, Phys. Rev. E 96 (2017), p. 012318.

[18] L. Lacasa, B. Luque, F. Ballesteros, J. Luque, and J. Nuño, From time series to complex networks: the visibility graph, Proc. Natl. Acad. Sci. USA 105 (2008), pp. 4972-4975.

[19] C. Lizama, $l_{p}$-maximal regularity for fractional difference equations on UMD spaces, Math. Nachr. 288 (2015), pp. 2079-2092.

[20] C. Lizama, The Poisson distribution, abstract fractional difference equations, and stability, Proc. Amer. Math. Soc. 145 (2017), pp. 3809-3827.

[21] B. Luque, L. Lacasa, F. Ballesteros, and J. Luque, Horizontal visibility graphs: Exact results for random time series, Phys. Rev. E 80 (2009), p. 046103.

[22] B. Luque, L. Lacasa, F. Ballesteros, and A. Robledo, Feigenbaum graphs: A complex network perspective of chaos, PLoS ONE 6 (2011), pp. 1-8.

[23] B. Luque, L. Lacasa, and A. Robledo, Feigenbaum graphs at the onset of chaos, Phys. Lett. A 376 (2012), pp. 3625-3629.

[24] R. May, Simple mathematical models with very complicated dynamics, Nature 261 (1976), pp. 459-467.

[25] K. Miller and B. Ross, Fractional difference calculus, in Univalent functions, fractional calculus, and their applications (Kōriyama, 1988), Ellis Horwood Ser. Math. Appl., Horwood, Chichester, 1989, pp. 139-152.

[26] A.M. Núnez, B. Luque, L. Lacasa, J.P. Gómez, and A. Robledo, Horizontal visibility graphs generated by type-I intermittency, Phys. Rev. E 87 (2013), p. 052801.

[27] M.G. Ravetti, L.C. Carpi, B.A. Gonçalves, A.C. Frery, and O.A. Rosso, Distinguishing noise from chaos: Objective versus subjective criteria using horizontal visibility graph, PLoS ONE 9 (2014), pp. 1-15.

[28] A. Robledo, Generalized statistical mechanics at the onset of chaos, Entropy 15 (2013), pp. $5178-5222$.

[29] C. Shannon, A mathematical theory of communication. The Bell Systems Tech. J. 27 (1948), pp. 379-423.

[30] C. Song, S. Havlin, and H.A. Makse, Origins of fractality in the growth of complex net- 
works, Nat. Phys. 2 (2006), p. 275.

[31] S. Strogatz, Nonlinear dynamics and chaos: with applications to physics, biology, chemistry, and engineering, Westview Press (2014).

[32] J. West, L. Lacasa, S. Severini, and A. Teschendorff, Approximate entropy of network parameters, Phys. Rev. E 85 (2012), p. 046111.

[33] G. Wu and D. Baleanu, Discrete chaos in fractional delayed logistic maps, Nonlinear Dynam. 80 (2015), pp. 1697-1703.

[34] G. Wu and D. Baleanu, Discrete fractional logistic map and its chaos, Nonlinear Dynam. 75 (2014), pp. 283-287.

[35] J. Zhang and M. Small, Complex network from pseudoperiodic time series: Topology versus dynamics, Phys. Rev. Lett. 96 (2006), p. 238701.

[36] A. Zygmund, Trigonometric series. Vol. I, II, Cambridge University Press, CambridgeNew York-Melbourne, 1977, Reprinting of the 1968 version of the second edition with Volumes I and II bound together. 